\title{
COMPENSATION FOR CHAIN GEARING ERRORS BY LONGITUDINAL MODIFICATION OF THE SPROCKET TEETH
}

\author{
Mamasobirov U. M.1, Shoobidov Sh. A. ${ }^{1}$, Kovalevskiy V.I. ${ }^{2}$ \\ ${ }^{1}$ Tashkent State Technical University \\ Email: red engineer94@mail.ru
}

\begin{abstract}
The article discusses the results of research work on the study of compensation for errors in the gearing in a chain transmission. The main indicator of the quality of the engagement is the completeness of the longitudinal contact. This indicator depends on the errors of the mutual position of the contacting surfaces for reasons of technological and deformative origin. Methods of compensation of errors of meshing in a chain transmission by a longitudinal modification of the sprocket teeth are described. The analysis of the research results showed that the localization of the contact by the method of longitudinal modification of the sprocket teeth can significantly improve the quality of the transmission by reducing the high sensitivity of the chain transmission to inaccuracies in the manufacture and installation of its elements.
\end{abstract}

Keywords: Compensation, Error, Gearing, Chain Drive, Sprocket Teeth, Quality Indicator, Gear Ring.

\section{Introduction}

The efficiency of gearing gears is largely determined by the completeness of the longitudinal contact - the main indicator of the quality of the gearing. This indicator depends on the errors in the mutual position of the contacting surfaces for reasons of technological and deformative origin. Technological errors like their manifestation can be attributed to one of two groups. The first group includes errors that do not depend on the frequency of rotation, the second - errors that change from tooth to tooth when the gear or sprocket rotates.

Errors of the first group are variable due to the random nature of their formation (appear from product to product), the second - due to their kinematic origin (appear at each revolution of each sprocket). Errors of deformative origin arise as a result of elastic deformations of structural transmission elements under load. The deformation values depend on the load of the gear and are therefore also variable.

In chain drives and chain conveyors, technological errors include:

Errors in the manufacture of elements of housings and supports and sampling of gaps in bearings, which lead to installation errors deviations in the relative position of the stars;

Errors in the manufacture of elements and assembly of chain links, which lead to nonparallelism and misalignment of the axes of the rollers; Errors in the shape of the rollers (bushings) of the chain and the transverse profile of the sprocket teeth;

Face runout of the sprocket ring gear concerning the operational axis of its rotation. The source of this error is the end runout of the workpiece on a gearcutting machine due to errors in basing when cutting teeth;

Face runout of the sprocket due to errors in locating on the shaft - installation error.

The face runout of the gear rim leads to a deterioration in the main indicator of the quality of engagement - the size, shape, and position of the contact spot, which vary from tooth to tooth when the sprocket rotates. In this case, the contact patch moves from one end to another in one revolution, and additional step errors associated with the end runout of the gear rim appear twice in one revolution. This defect can be practically eliminated or reduced on the sprockets themselves when installing a transmission in which composite sprockets with a special compensating device are used. If solid sprockets are used in the chain drive, then the harmful consequences of this defect can be prevented or significantly reduced when cutting teeth, including using their longitudinal modification.

Since the skew angles acting in the chain engagement are variable not only in value but also in direction, it is impossible to effectively use the method of rectilinear correction of the direction of the sprocket tooth to reduce them. Here it is advisable to use a more universal method of improving the quality of engagement - a longitudinal 
modification of the teeth, which has shown itself well in gear drives.

\section{Research Method}

One of the main tasks of the longitudinal modification of the teeth is to prevent the initial contact of the meshing elements at the end of the tooth. The task is to link the parameters of the modification with the errors in the relative position of the roller (sleeve) of the chain and the tooth of the sprocket. The choice of the required radius of curvature $\rho 0$ of the longitudinal profile of the tooth should, in the presence of manufacturing and installation errors and elastic deformations of the transmission elements, proceed from the requirements:

Contact strength of the tooth, which is a criterion for the performance of the sprocket in terms of wear resistance;

No displacement of the initial contact to the end of the tooth (edge contact), since such displacement is the cause of the concentration of the load in the joints of the hinge and its uneven distribution between the plates of the chain links.

The larger the radius of longitudinal curvature $\rho_{0}$, the lower the level of contact stresses in the engagement; therefore, it is desirable to strive for a larger value of $\rho_{0}$. An increase in $\rho_{0}$ worsens the localization of the contact, therefore, errors and elastic deformations of the elements of the chain drive or device can lead to edge contact. In turn, this leads to uneven distribution of specific pressures in the hinge, the load between the chainplates, and the load between the corresponding interfaces of the plates with bushings and rollers. From these considerations, it is desirable to strive for a decrease in $\rho_{0}$.

The lack of a theoretical justification for calculating the rational value of $\rho_{0}$ is one of the reasons why the longitudinal modification of the sprocket teeth is practically not used to improve the quality of chain drives. It should be noted that GOST 592 in transporting devices allows the manufacture of sprockets with a barrel-shaped tooth for plate chains when installed with an offset. At angles of conditional displacement $\varphi_{c}=3 \ldots 10^{\circ}$ in transporting devices, the recommended radius of curvature of the barrel-shaped surface

$$
\rho_{0}=\frac{28,65 b_{\mathrm{BH}}}{\varphi_{c}}
$$

where $b_{\mathrm{BH}}$ - the distance between inner plates.

The links of the chain on such teeth are selfaligning, due to which it is possible to reduce the concentration of stresses and specific pressures in the hinge and increase the performance of the chain.
A barrel-shaped tooth is obtained by cutting using a tool offset that smoothly changes from zero in the middle of the tooth to minus at its ends. The degree of barrel shape is assigned depending on the skew angle in the gear engagement. The absence of angular (edge) contact in barrel-shaped teeth is accompanied by an initial concentration of the load caused by their longitudinal curvature. In a working transmission, the sprocket tooth and the roller (bushing) roll mutually in the longitudinal direction, the contact patch moves towards one of the ends of the sprocket teeth. Simultaneously with an increase in the load and, consequently, the contact deformation of the engaging elements, the dimensions of the contact patch and the active length of the contact pads increase.

Here we consider the problem of choosing a rational value based on the requirements for the contact strength of the sprocket teeth. That is, it is determined from only one condition $l_{\mathrm{M}}=\Pi b$ - the length of the contact spot for the required localization of $\Pi$.

Let us estimate the effect of the curvature of the generatrix of the tooth on the nature of the change in the gap between the tooth and the roller (bushing), on the error in the position of the engaging elements, and on the displacement of the point of initial contact in the presence of errors and elastic deformations of the structural elements of the chain drive or chain device. In work [1], the equation for changing the clearance between the teeth of the gear transmission along their rectilinear generators is given. Similarly, the gap between the sprocket tooth and the roller (bushing) changes along their straight generatrices, therefore, it is characterized by the equation

$\Delta \mathrm{z}(\mathrm{x}, 0)=\Delta \mathrm{z}_{1}+\Delta \mathrm{z}_{2}+\mathrm{x}\left(\Delta \mathrm{\gamma}_{\mathrm{y} 1}+\Delta \mathrm{\gamma}_{\mathrm{y} 2}\right)$

Where, $\Delta z_{1}+\Delta z_{2}=\Delta z_{0}$ represents a constant component, and the last term $x\left(\Delta \gamma_{y_{1}}+\Delta \gamma_{y_{2}}\right)-$ is a variable component of the lumen along the length of the tooth of the sprocket, due to the primary transmission errors.

The constant component is the roller position error equivalent to the rotation error of the driven sprocket and does not affect the load distribution along the tooth length. The variable component characterizes the distribution of the gap between the tooth and the roller (sleeve) along their length and is an objective characteristic of the longitudinal contact error.

\section{Obtained Scientific Results and their Analysis}

The longitudinal modification of the sprocket teeth introduces an additional component into the 
angular clearance in the cross-section normal to the tooth profile, equivalent to the clearance, which is formed by the barrel shape (Fig. 1)

$$
\Delta z_{\rho}^{\prime} \approx \frac{x^{2}}{2 \rho_{0}}
$$

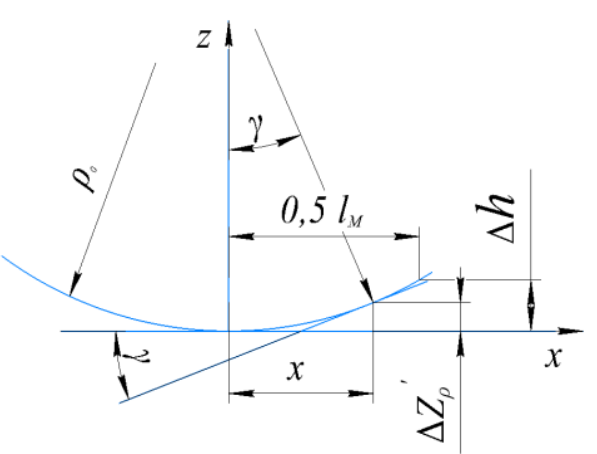

Figure 1: Clearance in engagement with longitudinal modification of sprocket teeth

Taking into account this component, the lumen variable along the length of the sprocket tooth along the line of initial contact will be distributed according to the contact pattern of the tooth and roller (Fig. 2) according to the dependence

$$
\begin{aligned}
& \Delta z(x, 0)=0,5 b+x\left(\Delta \gamma_{y 1}+\Delta \gamma_{y 2}\right)+ \\
& +\frac{x^{2}}{2 \rho_{0} \cos \psi \cos \left(\Delta \gamma_{y 1}+\Delta \gamma_{y 2}\right)}
\end{aligned}
$$

where $\psi=(2 \pi / z)+\varphi-$ pressure angle not shown in the diagram; $z$ - number of sprocket teeth.

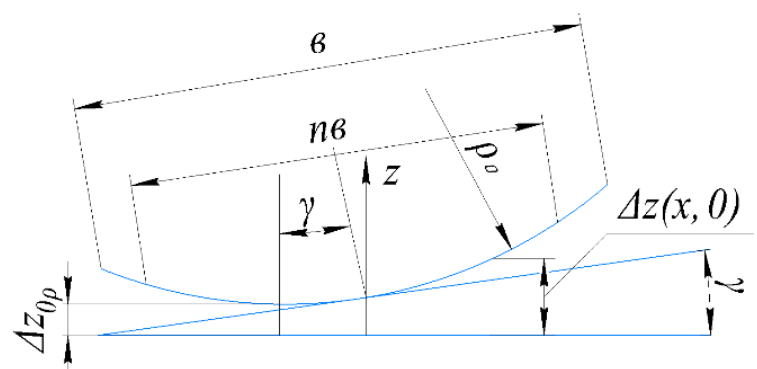

Figure 2: Parameters of contact of the tooth with the longitudinal modification and the roller of the chain drive

The barrel-shaped tooth due to the displacement of the point of initial contact to the middle of the tooth will cause an additional component of constant clearance - the error in the position of the roller (sleeve) is equivalent to the error in the rotation of the driven sprocket

$$
\Delta z_{0} \rho(x, 0)=0,5 b\left(\Delta \gamma_{y 1}+\Delta \gamma_{y 2}\right)-\frac{x_{*}^{2}}{2 \rho_{0} \cos \varphi}
$$

where $0,5 b$ - coordinate of the point of initial contact of the roller and the tooth with a straight generatrix, counted from the middle section of the gear rim; $x_{\text {s }}$ - the same with longitudinal modification of the tooth.

For teeth with a straight generatrix, the coordinate of the point with the minimum clearance is always known, since this point is located at one of the ends. To find the coordinate of a point with a minimum clearance for teeth with a longitudinal modification, it is required to find the extremum of function (4), i.e. fulfill the condition:

$$
\frac{\partial(\Delta z(x, 0))}{\partial x}=0
$$

The solution to this problem will make it possible to assess the sensitivity of the chain engagement in the transmission with a modified sprocket tooth to errors in the mutual position of the contacting elements and to determine the rational size of the radius of the longitudinal curvature of the tooth $\rho_{0}$.

Differentiating equation (4) and fulfilling condition (6), we obtain

$$
\begin{aligned}
& \frac{\partial(\Delta z(x, 0))}{\partial x}=\Delta \gamma_{y 1}+\Delta \gamma_{y 2}+ \\
& +\frac{x}{\rho_{0} \cos \psi \cos \left(\Delta \gamma_{y 1}+\Delta \gamma_{y 2}\right)}=0
\end{aligned},
$$

where the sought coordinate of the initial contact of the barrel-shaped tooth

$$
x_{*}=\left(\Delta \gamma_{y 1}+\Delta \gamma_{y 2}\right) \rho_{0} \cos \psi=\Delta \gamma_{y} \rho_{0} \cos \Delta \gamma_{y}(7)
$$

Thus, a linear relationship was found between the reduced mounting error $\Delta \gamma_{y}=\left(\Delta \gamma_{y 1}+\Delta \gamma_{y 2}\right) \cos \psi$ and the coordinate $x_{*}$ of the point of initial contact of the tooth with the longitudinal modification and the roller.

In real chain drives, $\Delta \gamma \leq 0.01 \mathrm{rad}$, in this regard, without a noticeable loss of accuracy, we take $\cos \Delta \gamma_{\mathrm{y}}$ $\approx 1$ in formula (7). Then

$$
x_{*}=\Delta \gamma_{y} \rho_{0}
$$

The contact area in the presence of a mounting error $\Delta \gamma_{y}$ will not go beyond the tooth length (Fig. 3) if the condition is met

$$
x_{*}+0,5 l_{\mathrm{M}} \leq 0,5 \Pi b
$$

where $0,5 \Pi b$ - permissible displacement of the extreme point of the contact patch, measured from the mean section of the tooth, and $l_{\mathrm{M}}-$ contact pad length. 
The dependence of the longitudinal curvature of the sprocket tooth on the given localization of the contact area $\Delta h$ follows from Fig. 1

$$
\frac{1}{\rho_{0}}=\frac{2 \Delta h}{\left(0,5 l_{\mathrm{M}}\right)^{2}}, \Delta h=\frac{\left(0,5 l_{\mathrm{M}}\right)^{2}}{2 \rho_{0}},
$$

where $0,5 l_{\mathrm{M}}=\left(2 \Delta h \rho_{0}\right)^{1 / 2}$.

From condition (8), taking into account $(7, a)$ and (9), we have

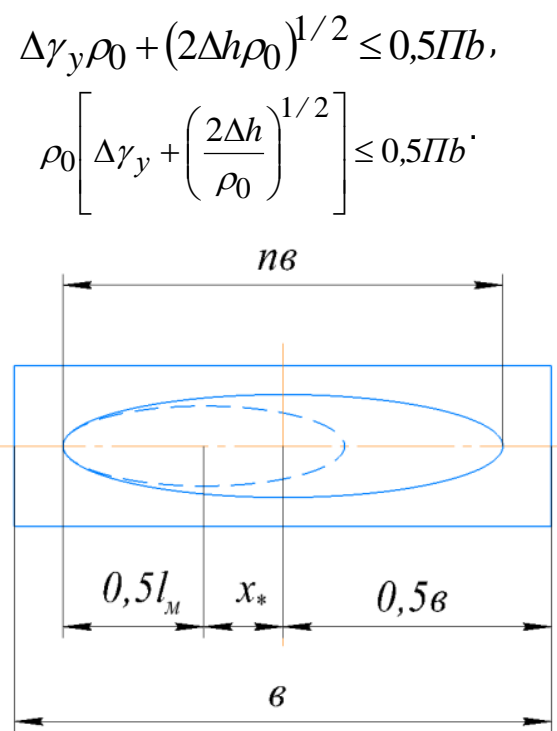

Figure 3: Contact spot parameters

By the method of successive approximations, let us get rid of the right side of the last expression in the denominator.

As a first approximation, we take:

$$
\rho_{0(1)}=\frac{0,5 \Pi b}{\Delta \gamma_{y}}
$$

In the second approximation, we finally obtain with sufficient accuracy the calculated dependence in the explicit form:

$$
\rho_{0}=\frac{0,5 \Pi b}{\Delta \gamma_{y}\left[1+\left(\frac{2 \Delta h}{0,5 \Pi b \Delta \gamma_{y}}\right)^{1 / 2}\right]}
$$

Formula (10) determines the value of the radius of the longitudinal curvature of the teeth of the sprocket $\rho_{0}$, at which the extreme point of the contact spot will be removed from the middle of the section of the tooth by a distance of $0.5 \Pi \mathrm{b}$, depending on the localization of the contact area $(\Delta \mathrm{h})$ and installation errors $\left(\Delta \gamma_{\mathrm{y}}\right)$ - by the value load parameter $\mathrm{b} \Delta \gamma_{\mathrm{y}} / \Delta \mathrm{h}$. This formula takes into account only the geometry of the contacting surfaces of the elements of the chain engagement.
It does not show that $\rho_{0}$ depends on the transmission load, since the reduced error $\Delta \gamma_{\mathrm{y}}$ is the technological component of the total error

$$
\Delta \gamma_{y}^{\prime}=\Delta \gamma_{y}+\Delta \gamma_{\text {удеф }},
$$

where $\Delta \gamma_{\text {удеф }}$ - deformative component associated with transmission load.

Using work [1], one can, for example, determine the admissible value of the radius of longitudinal curvature from the condition

$$
l_{\mathrm{M}}=\Pi^{\prime} b
$$

By substituting in (11) the values of the length of the contact area, found by solving the problem of contact of two elastic bodies with finite radii of curvature in two directions (Hertz's solution), the ratio of the radii was obtained at which the given degree of localization is provided

$$
\frac{\rho}{\rho_{0}}=\left(\frac{1,465}{\Pi^{\prime}}\right)^{8 / 3}\left(\frac{F_{n} \rho}{b^{3} E}\right)^{8 / 9}
$$

where $\rho$ - reduced radius of curvature of the tooth and roller in the transverse direction, $E$ - reduced modulus of elasticity of roller and sprocket materials.

It is known that each chain pivot is located on the working part of the sprocket tooth profile from the beginning of the engagement and almost until the very disengagement. The contact stresses in the tooth from the static application of the load are determined by the normal pressure between the contacting surfaces of the chain joint bodies and the sprocket tooth. It is also known that the normal pressure in the most loaded hinge changes from zero at the initial moment of engagement to the highest one when turning around the sprocket rotation axis by an angle of $2 \pi / z$ at the end of the engagement

$$
F_{n}=S_{\mathbf{B}} \frac{\sin \frac{2 \pi}{z}}{\sin \psi}
$$

where $S_{\mathrm{B}}$ - tension force of the driving chain.

In the course of long-term operation of the chain transmission, the contact of the tooth and the roller occurs on the concave and transitional section of the profile at the beginning of the operation, then on the rectilinear and the convex section during the last period of operation. In this regard, for an asterisk by GOST 591, the reduced radii of curvature of the tooth and roller are determined by the curvatures of the corresponding sections of the lateral profile: $\rho^{-1}=r_{\mathrm{p}}^{-1}-r_{1}^{-1}-$ on location $E F ; \rho^{-1}=\rho_{\mathrm{p}}^{-1}-$ on 
location $F G ; \mathrm{p}^{-1}=r_{\mathrm{p}}^{-1}+r_{2}^{-1}-$ on location $G K$. Here $r_{\mathrm{p}}$ is the radius of the roller (bushings), $r_{1}$ - radius of the transition area of the tooth $\mathrm{EF}, r_{2}$ - the same convex FG. The maximum contact stresses according to $\mathrm{G}$. Hertz when the cylinders are in contact will be on the convex section of the tooth, for which the reduced radius of the transverse curvature in formula (12) should be determined by $r_{2}$ of the standard profile

$$
\rho=\frac{r_{\mathbf{p}} r_{2}}{r_{2}+r_{\mathbf{p}}}
$$

The most favorable from the point of view of the operability of the chain transmission can be considered the contact of the roller and the tooth on the transition curve of the profile (in the section EF with the radius of curvature $r_{1}$ ), i.e. in the area of normal engagement. The reduced radius of curvature for such contact is determined by the radius $r_{1}$ of the standard profile

$$
\rho=\frac{r_{\mathbf{p}} r_{1}}{r_{1}-r_{\mathbf{p}}}
$$

As a result of the study of contact stresses in gears with engagement with localized contact of the teeth [1], the dependence for the radius of longitudinal curvature, at which the maximum effect of longitudinal modification of the teeth is achieved

$$
\rho_{0}=0,2355 \frac{b}{\gamma}
$$

It follows from the formula that the rational value of $\rho_{0}$ is determined only by the geometric (b) and accuracy $(\gamma)$ parameters.

Using relationship (15) and formula (12), we find the required contact localization at which the maximum effect of longitudinal modification of the sprocket teeth is achieved.

After substituting (15) into (12), taking $\gamma=\Delta \gamma_{y}$, we obtain the expression

$$
\frac{\rho \Delta \gamma}{b}=0,2355\left(\frac{1,465}{\Pi^{\prime}}\right)^{8 / 3}\left(\frac{F_{n} \rho}{b^{3} E}\right)^{8 / 9},
$$

from which the required contact localization is found,

$$
\Pi^{\prime}=0,852\left(\frac{b}{\rho \Delta \gamma_{y}}\right)^{3 / 8}\left(\frac{F_{n} \rho}{b^{3} E}\right)^{1 / 3}
$$

where $b / \rho \Delta \gamma_{y}$ - geometric, $F_{n} \rho / b^{3} E$ - load parameters.

The problem of reducing the maximum contact stresses in the engagement $\sigma_{\gamma}$ caused by errors in the manufacture and installation of the gear, by the method of longitudinal modification of the sprocket teeth by a factor of $M$, i.e. up to the value $\sigma=\sigma_{\gamma} / \mathrm{M}$, can be solved as follows. First, the admissible value of $\rho / \rho_{0}$ is found, for example, according to formulas (14), (14, a) and (15), after which, taking into account the condition $l_{\mathrm{M}}=\Pi^{\prime}$, the required localization $\Pi$ ' is found.

In [1], using appropriate substitutions and transformations, an expression was obtained that, taking into account the analogy of the contact interaction of surfaces in gear and chain gearing, can be applied to the chain transmission

$$
\Pi^{\prime}=1,465\left(\frac{M}{r}\right)^{1 / 5}\left(\frac{F_{n} \rho}{E b^{3}}\right)^{m}\left(\frac{b}{\rho \Delta \gamma_{y}}\right)
$$

For loads typical of chain transmission and total angles of misalignment $\Delta \gamma_{y}=\Delta \gamma_{y 1}+\Delta \gamma_{y 2}$ in the engagement, the load parameter expressing the requirement for the localization of the contact, $\Delta h / \rho \Delta \gamma_{y} \leq 0,5$ At such values of the load parameter according to [1] $r=0,941, m=0,683, n=0,06$.

To ensure a uniform distribution of specific pressures in the hinge and the load from the tension of the chain between its plates, it is impossible to allow the contact area to reach the end of the tooth and it is necessary to strive for the minimum value $x_{\text {s }}$ - the displacement from the middle of the tooth of the point of initial contact $(7, a)$. The solution of a similar problem for a gear train [1] gives an admissible ratio for the $0,5 l_{\mathrm{M}}=0,5 b-\Delta \gamma_{y} \rho_{0}$ condition.

$$
\text { e } \frac{\rho}{\rho_{0}}>17,578\left(\frac{F_{n} \rho}{b^{3} E}\right)^{8 / 9}
$$

The influence of localization of contact on the distribution of the load between the plates of the chain, let us estimate the coefficient of uneven distribution of the load from the tension of the leading branch of the chain between the plates of the link:

$$
K_{S}=\frac{S_{1}}{S_{2}}
$$

where $S_{1}, S_{2}$ - link plate forces. 
The displacement of the point of initial contact between the profiles of the barrel-shaped tooth and the roller (Fig. 4) will lead to a displacement of the line of action of the chain tension relative to the longitudinal axis of the chain by the value $x *$.

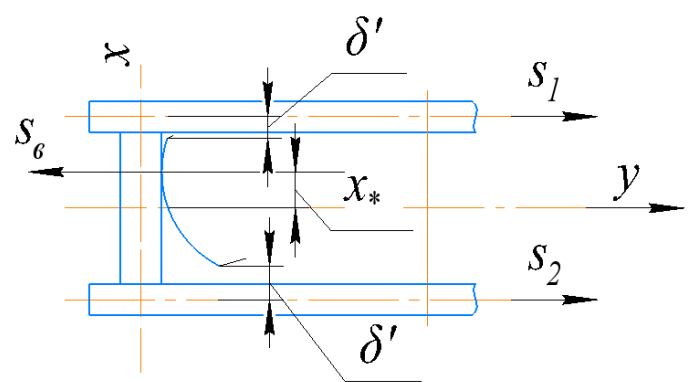

Figure 4: Diagram of the distribution of forces in the lamellar chain.

From the equilibrium conditions of the link under the action of forces

$$
S_{6} x_{*}-0,5\left(b+\delta^{\prime}\right)\left(S_{1}-S_{2}\right)=0,-S_{\theta}+S_{1}+S_{2}=0
$$

Should

$$
\frac{\left(S_{1}+S_{2}\right) x_{*}}{0,5 b}-\left(S_{1}-S_{2}\right)-\frac{\delta^{\prime}}{b}\left(S_{1}-S_{2}\right)=0
$$

In the resulting expression, let us take $x_{*} / 0,5 b=K_{\rho}$ - the coefficient of the relative displacement of the contact area caused by the barrel-shaped tooth of the sprocket, and take it into account (18). After the appropriate substitutions and transformations, we obtain the coefficient

$$
K_{\rho}=\frac{K_{S}-1}{K_{S}+1}\left(1+\frac{\delta^{\prime}}{b}\right)
$$

expressing the relationship between the position of the contact patch relative to the middle of the roller, the requirement for equal distribution of the load between the plates $\left(K_{\mathrm{s}}\right)$, and the design factor $\left(\left(\delta^{v} / b\right)\right.$.

\section{Conclusions}

Based on the studies, the following conclusions can be drawn:

1. The possibility of obtaining metal layered compositions of the Mo-TiC system has been established. This composition allows the manufacture of cutting and stamping tools by casting on gasified models.

2. The technology of thermocyclic processing (TCP) has been developed during which structures are formed with a maximum of crystal structure defectiveness.

3. As a quenching liquid, instead of oil, it is possible to use the water-soluble preparation "Uniflock" produced in Uzbekistan.

4. Sintered antifriction cermet alloys obtained from local raw materials, both in terms of mechanical and antifriction properties, meet the requirements of technical conditions.

5 . The optimal composition of the charge in the production of ceramic-metal alloys of antifriction purpose is: $97 \%$ iron powder, $2 \%$ elemental or pencil graphite, and $1 \%$ pyrite.

\section{Acknowledgements}

The authors would like to acknowledge Professors of the "Mechanical engineering" department of Tashkent State Technical University, Ministry of Higher Education Uzbekistan, and mechanics of the "Materials Science" department.

\section{References}

[1] Norkhudjaev F.R., Mavlonova S.F. Improving the wear resistance and durability of plowshares of longline plows for tillage // Composite materials, 2016. - №. 1.- P. 70 -74. (05.00.00 №13).

[2] Norknudznaev F. R., Nazarov A. M., Yakubov L. E. Sintered powder composition based on Mo - TiC. India. International Journal of Advanced Research in Science, Engineering, and Technology, 2016. Vol.3, Issue 7, July. pp. 2347-2350. (05.00.00 №8).

http://www.ijarset.com/upload/2016/july/2 IJARS ET_yahov_latest.pdf

[3] Norknudznaev F. R., Nazarov A. M., Koveshnikov S. V., Mavlonov Sh. A., Khurbanbaev Sh. Z., Ataullaev A. O. The fiber-optical sensor for chromate-graphic measurements. India. International Journal of Advanced Research in Science, Engineering, and Technology, 2016. Vol.3, Issue 8, August. pp. 2463-2467. (05.00.00 №8).

[4] Norknudznaev F. R., Yakubov L. E. Actual prospects of application of type "Fss - an intermediate alloy - solid working element" in the manufacture of drill tools. Austria. 7 - 8 (July August). - pp. 176 - 178. (05.00.00 №3).

[5] Norkudjayev F.R., Alikulov A. Kh. Abdurakhmonov Kh. Z. Tursunov T. Kh. Examination of Thermophysical Processes in the Creation of Metal Layered Compositions. International Journal of Recent Technology and Engineering (IJRTE) ISSN: 2277-3878, Volume-8 Issue-2S10, September 2019. P 361-366.

https://www.scopus.com/record/display.uri?eid=2s2.0- 
85075285756\&origin=AuthorNamesList\&txGid= 5e9426dea9148b5d4943f35d019901c5

[6] Fedyukin V.K. Method of thermocyclic metal processing. - L.: LSU, 1984.-190p.

[7] Fedyukin V.K. Termociklicheskaya obrabotka: tehnologiya, struktura i svoistva metallicheskih materialov [Thermal cycling: technology, structure and properties of metallic materials]. Ins-t problem mashinovedeniya ANSSSO, 1991. $309 \mathrm{~s}$.

[8] Shakirov Sh.M., Bektemirov B.Sh., Alimbabaeva Z.L., Abdurakhmonov Kh.Z., Tursunov T.Kh. Formation of Structure at Thermodiffusion Chroming of Porous Permeable Materials Based on Iron Powder. Test Engineering and Management. ISSN: 0193-4120, Volume 83 Issue - March/April 2020, 12 March 2020. P 635-638.

http://testmagzine.biz/index.php/testmagzine/artic $\underline{\text { le/view/3626 }}$
[9] Norkudjayev F.R., Mukhamedov .A.A., Khudayberdiev .O.R., Ergashev D.M., Djalolova S.T. Technological capabilities of application of thermocyclic processing (tcp) tool steel. International Journal of Psychosocial Rehabilitation. ISSN: 1475-7192, Volume-24 Issue 08, April 2020. p1866-1874.

https://www.psychosocial.com/article/PR280207/ $16691 /$

[10] German R.M.Powde Metallurgy and Particulate Materials Processing, Metall Powder Industries Federation, Princeton, NJ 2005.-P 308.

[11] Norkudjayev F.R. Mukhamedov A.A. Xudayberdiyev O.R. Receving antifuction materials based on the waste of metallurgical Productions of Uzbekistan. International Journal of Advanced Research in Engineering and Technology (IJARET) NJ 2019.-P 8675-8677. 